\title{
Generation of Binary Sequences of Length 10230 Bits Having Better Odd and Even Correlation with Large Linear Complexity for Use in Global Navigation Satellites Systems (GNSS) Applications
}

\author{
Dileep Dharmappa ${ }^{1 *}$, Mahalinga V. Mandi², Ramesh Siddaiah ${ }^{3}$ \\ ${ }^{1}$ Department of Electronics and Communication Engineering (ECE), Sri Siddhartha Academy of Higher Education (SSAHE), \\ Agalakote, Tumakuru 572107, Karnataka, India \\ ${ }^{2}$ Navigation Systems Area, ISRO Telemetry Tracking and Command Network (ISTRAC), ISRO, Bengaluru 560058, \\ Karnataka, India \\ ${ }^{3}$ Department of Electronics and Communication Engineering (ECE), Dr. Ambedkar Institute of Technology, Near Jnana \\ Bharathi, Bengaluru 560056, Karnataka, India
}

Corresponding Author Email: dileep@istrac.gov.in

https://doi.org/10.18280/mmep.070112

Received: 2 September 2018

Accepted: 2 January 2020

\section{Keywords:}

Global Positioning System (GPS), Global Navigation Satellites Systems (GNSS), chaotic logistic map, auto correlation, Cross Correlation, Linear Complexity (LC)

\begin{abstract}
Binary sequences used in Global Navigation Satellites Systems (GNSS) are found to be easily decoded due to less linear complexity. The correlation properties are extremely important while selecting the sequences for GNSS Applications. Linear Feedback Shift Register (LFSR) based Sequences are being used in GNSS Systems such as Global Positioning System (GPS) L2 CM (L2 frequency band Civil Moderate) Signal. Due to the short cycling of LFSR these sequences result in poor correlation properties. In this work the properties of binary sequences used in the state of the art GPS L2CM Navigation signal is explored. The odd and even correlation properties of GPS L2CM sequences are presented in detail. These sequences are analyzed for their Linear Complexity property. A method is proposed for generation of 10230 bit length binary sequences. A new set of binary sequences with set size of 47 sequences are generated using chaotic real sequences. The generated sequences are analyzed for odd correlation, even correlation and linear complexity properties. The proposed binary sequences are found to have better correlation and excellent linear complexity properties as compared to GPS L2 CM Sequences, which make them suitable for use in GNSS Applications.
\end{abstract}

\section{INTRODUCTION}

Binary sequences play vital role in Global Navigation Satellites Systems (GNSS) Systems. GNSS Systems such as Global Positioning System (GPS) by US, European GNSS (GALILEO), Russians Global Navigation Satellite System (GLONASS), Indian Regional Navigation Satellite System (IRNSS) by India, Quasi Zenith Satellite System (QZSS) by Japan and Chinese BeiDou Navigation Satellite System (BEIDOU/COMPASS) use binary sequences for various types of services provided by each GNSS system. User receiver at ground identifies a unique service from a GNSS System using these binary sequences.

GPS system is widely used for location services across the world. Towards enhancing the capabilities of GPS system, a new GPS signal namely GPS L2 band Civil Moderate (GPS L2 CM) signal having binary sequences of length 10230 bits was added in L2 frequency. This signal provides one order better accuracy than the existing GPS C/A (Coarse Acquisition) signal having binary sequences of length 1023 bits.

Binary spreading sequences provide secure and reliable transmission for GNSS Systems. Binary sequences derived from Linear Feedback Shift Register (LFSR) are the commonly used Pseudo Random Binary Sequences in spread spectrum applications [1]. For GNSS Systems, the property of spreading sequence length plays an important role. It is always designed such that the spreading sequence length should be an integer multiple of chip rate. However binary sequences generated using conventional methods such as Gold Codes, which are based on LFSR are restricted to code length $\left(2^{\mathrm{N}}-1\right)$ for ' $N$ ' stages. The GPS L2 CM sequences are generated using $\mathrm{N}=27$ stage LFSR. The length of the binary sequence of 27 stage LFSR is 134217727 bits. However to meet the sequence length constraints these binary sequences are short cycled/reset to 10230 bits in state-of-the art GPS Navigation System. The process of short cycling results in poor correlation properties of the truncated sequences [1]. The desirable properties to be considered in the design of the binary sequences for GNSS Systems are described in the literature [1-5]. However in the existing literature, the detailed analysis of the properties namely odd correlation and linear complexity of the new civilian signal GPS L2 CM sequences are not done.

The security of the GNSS Systems depends on the linear complexity property of the binary spreading sequences. It is observed from the literature that due to the low linear complexity property binary sequences used in BeiDou System were decoded by Gao et al. [6]. Also similar instance of decoding Galileo In Orbit Validation Element-A (GIOVE-A) codes, was done by Psiaki et al. [7]. Design of binary sequences with high linear complexity is being attempted by many researchers [8-11]. 
Binary sequences used for GNSS Applications are expected to perform well in real world environment such as multipath, interference etc. Whenever there is a flip in a bit in the navigation sequence or the bit in the secondary code sequence is observed within the integration period of the binary spreading sequence, it is difficult to acquire the signal and this depends on the odd correlation properties of the spreading sequence [12-14].

In this work a method is proposed to derive binary sequences with good even correlation, odd correlation and linear complexity properties. Proposed binary sequences are of length 10230 bits and are derived from chaotic real valued sequences.

The paper is organized as follows. Correlation and linear complexity properties are defined in the Section 2. Section 3 provides correlation and linear complexity properties of GPS L2 CM sequences in detail. In Section 4, proposed method of generating chaotic binary sequence is discussed. The correlation and linear complexity properties of the proposed binary sequences are provided in Section 5. Section 6 concludes the work.

\section{CORRELATION AND LINEAR COMPLEXITY PROPERTIES}

In this section the correlation and linear complexity properties are defined.

\subsection{Correlation property}

Considering two binary sequences $\mathrm{x}$ and $\mathrm{y}$ of length $\mathrm{N}$ bits the aperiodic correlation is defined [15] and is given by,

$$
C_{x, y}(l)=\left\{\begin{array}{lr}
\sum_{j=0}^{N-1-l} x_{j} y_{j+l}^{*}, & 0 \leq l \leq N-1 \\
\sum_{j=0}^{N-1+l} x_{j-l} y_{j}^{*}, & 1-N \leq l \leq 0 \\
0, & |l| \geq N .
\end{array}\right.
$$

Here $l$ denotes the shift of one sequence say y relative to the other sequence $\mathrm{x}$.

Even Cross Correlation (even CCR) property between two sequences $\mathrm{x}$ and $\mathrm{y}$ is provided [15] by,

$$
\theta_{x, y}(l)=C_{x, y}(l)+C_{x, y}(l-N)
$$

Even Auto Correlation (even ACR) property for a single sequence say, sequence $\mathrm{x}$ or sequence $\mathrm{y}$ is defined [15] as,

$$
\theta_{x, x}(l)=C_{x, x}(l)+C_{x, x}(l-N)
$$

Odd Cross Correlation (odd CCR) property between two sequences $\mathrm{x}$ and $\mathrm{y}$ is provided [15] by,

$$
\hat{\theta}_{x, y}(l)=C_{x, y}(l)-C_{x, y}(l-N)
$$

Odd Auto Correlation (odd ACR) property for a single sequence say, sequence $\mathrm{x}$ or sequence $\mathrm{y}$ is defined [15] as

$$
\hat{\theta}_{x, x}(l)=C_{x, x}(l)-C_{x, x}(l-N)
$$

The importance of properties of even correlation is well understood in the literature for GNSS systems. Any improvement in the correlation is an added advantage for GNSS system.

Especially the odd correlation plays an important role in GNSS systems when there is a flip in the bit of the binary sequence, due to the presence of multipath or interference and that leads to difficulty in acquiring and tracking the satellite signals.

\subsection{Linear complexity property}

Linear complexity [16] for a binary sequence represents the length of shortest LFSR required to generate the same binary sequence. Berlekamp - Massey algorithm [16] is commonly used for computation of Linear complexity.

Linear complexity property plays vital role in advanced applications of GNSS systems such as security of driverless cars, Unmanned Arial Vehicles (UAV), Internet of Things (IoT) etc.

Next section provides the correlation property and linear complexity property of 10230 bit GPS L2 CM binary sequences.

\section{CORRELATION AND LINEAR COMPLEXITY PROPERTIES OF GPS L2 CM SEQUENCES}

GPS L2 CM sequences of length 10230 bits are generated using the method provided in the GPS Interface Control Document (ICD) [17] and the correlation and linear complexity properties are analyzed.

\subsection{Generation of GPS L2 CM sequences}

The GPS L2 CM code is a new civilian signal which has improved navigation accuracy which repeats for every $20 \mathrm{~ms}$ and are LFSR based codes reset after 10230 bits having chip rate of is $511.5 \mathrm{Kbps}$.

These codes are being transmitted from block II series replenishment satellites (IIR-M), block II series follow on satellites (IIF) and also subsequent blocks of GPS Satellites. Block diagram of the GPS L2 CM signal generator is shown in Figure 1. The L2 CM sequences are obtained by short cycling the LFSR for every count of 10230 chips by resetting with a specified initial state. The polynomial used to generate the L2 CM signal is 1112225171 (octal) of degree 27 . The 27 bit LFSR provides sequences of length $\left(2^{27}-1\right)$ which is equal to 134217727 bits out of which 134207497 bits are discarded to get the required 10230 bit sequences. A set of 37 binary sequences of length 10230 bits are generated from the method given in Figure 1 by selecting different initial values for LFSR and all the 37 sequences are short cycled to 10230 bits. The initial values to generate the 37 sequences are given in Table 1 .

LFSR based sequences are most commonly used binary sequences for GNSS. However for GNSS Signals, it is desirable to have binary sequences period to be multiple of onboard fundamental frequency $(10.23 \mathrm{MHz})$ i.e. multiple of 1023. Considering $10^{\text {th }}$ multiple of 1023 bits which is equal to 10230 bits and is the required binary sequence length of GPS L2 CM sequences. However it is not possible to generate 
10230 bit period, binary sequences using LFSR. Hence the sequences from LFSR are truncated/short cycled to $10230 \mathrm{bits}$.
All the 37 GPS L2 CM sequences of length 10230 bits are generated using MATLAB.

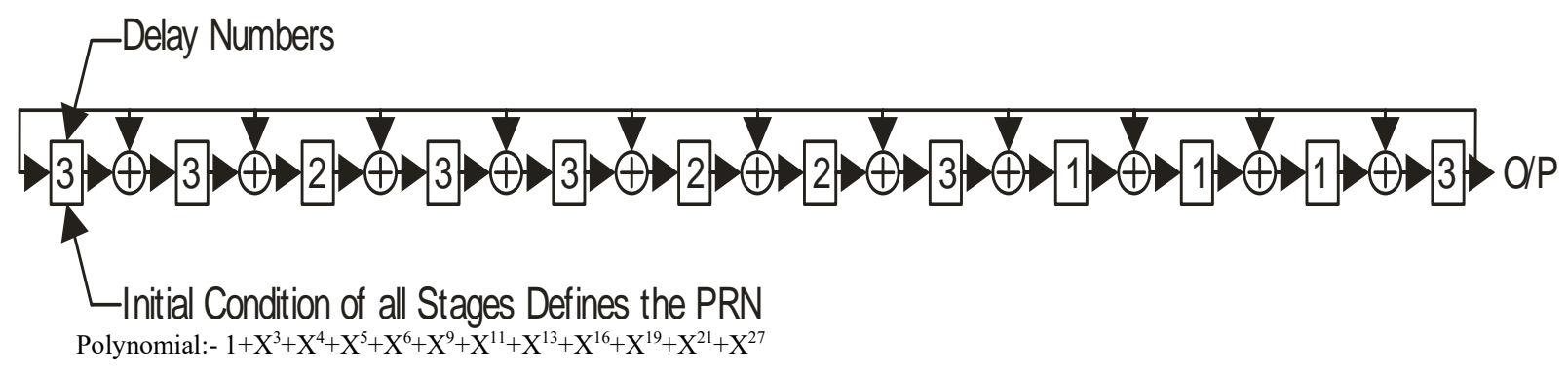

Figure 1. GPS L2 CM Binary Sequence generator

Table 1. Initial values of 27 stage LFSR for 37 GPS L2 CM sequences

\begin{tabular}{|c|c|c|c|}
\hline$S$ & GPS & Initial Shift & End Shift \\
\hline V & PRN No & register & register \\
\hline ID & & Stage(Octal) & Stage $($ Octal $)$ \\
\hline 1 & 1 & 742417664 & 552566002 \\
\hline 2 & 2 & 756014035 & 034445034 \\
\hline 3 & 3 & 002747144 & 723443711 \\
\hline 4 & 4 & 066265724 & 511222013 \\
\hline 5 & 5 & 601403471 & 463055213 \\
\hline 6 & 6 & 703232733 & 667044524 \\
\hline 7 & 7 & 124510070 & 652322653 \\
\hline 8 & 8 & 617316361 & 505703344 \\
\hline 9 & 9 & 047541621 & 520302775 \\
\hline 10 & 10 & 733031046 & 244205506 \\
\hline 11 & 11 & 713512145 & 236174002 \\
\hline 12 & 12 & 024437606 & 654305531 \\
\hline 13 & 13 & 021264003 & 435070571 \\
\hline 14 & 14 & 230655351 & 630431251 \\
\hline 15 & 15 & 001314400 & 234043417 \\
\hline 16 & 16 & 222021506 & 535540745 \\
\hline 17 & 17 & 540264026 & 043056734 \\
\hline 18 & 18 & 205521705 & 731304103 \\
\hline 19 & 19 & 064022144 & 412120105 \\
\hline 20 & 20 & 120161274 & 365636111 \\
\hline 21 & 21 & 044023533 & 143324657 \\
\hline 22 & 22 & 724744327 & 110766462 \\
\hline 23 & 23 & 045743577 & 602405203 \\
\hline 24 & 24 & 741201660 & 177735650 \\
\hline 25 & 25 & 700274134 & 630177560 \\
\hline 26 & 26 & 010247261 & 653467107 \\
\hline 27 & 27 & 713433445 & 406576630 \\
\hline 28 & 28 & 737324162 & 221777100 \\
\hline 29 & 29 & 311627434 & 773266673 \\
\hline 30 & 30 & 710452007 & 100010710 \\
\hline 31 & 31 & 722462133 & 431037132 \\
\hline 32 & 32 & 050172213 & 624127475 \\
\hline 33 & $*$ & 500653703 & 154624012 \\
\hline 34 & $*$ & 755077436 & 275636742 \\
\hline 35 & $*$ & 136717361 & 644341556 \\
\hline 36 & $*$ & 756675453 & 514260662 \\
\hline 37 & $*$ & 435506112 & 133501670 \\
\hline
\end{tabular}

\subsection{Even and odd ACR properties of GPS L2 CM sequences}

Investigation is carried out for even and odd ACR properties. The results of maximum off peak even ACR and maximum off peak odd ACR values are tabulated in Table 2. The off peak even and odd ACR values are found to be multiple valued because of truncation. From Table 2, it is found that maximum even ACR for the set of 37 sequences is $-26.90 \mathrm{~dB}$ and the maximum odd ACR is found to be $-27.24 \mathrm{~dB}$. The histogram of off peak even ACR and the off peak odd ACR is shown in Figure 2 and Figure 3 respectively. In Figure 2, it is found that only for 5 sequences out of 37 sequences are having off peak even ACR value of $-29.2 \mathrm{~dB}$. In Figure 3, it is found that only for 4 sequences out of 37 sequences are having off peak odd ACR value of $-28.5 \mathrm{~dB}$.

Table 2. Off peak maximum even ACR and odd ACR for 37 GPS L2 CM sequences

\begin{tabular}{|c|c|c|}
\hline $\begin{array}{c}\text { GPS L2 CM } \\
\text { Sequence } \\
\text { Number }\end{array}$ & $\begin{array}{c}\text { Off Peak } \\
\text { Maximum } \\
\text { even ACR } \\
\text { Value }\end{array}$ & $\begin{array}{l}\text { Off Peak Maximum } \\
\text { odd ACR Value }\end{array}$ \\
\hline 1 & -28.833 & -27.408 \\
\hline 2 & -28.928 & -28.602 \\
\hline 3 & -28.928 & -28.070 \\
\hline 4 & -28.833 & -27.569 \\
\hline 5 & -28.466 & -29.120 \\
\hline 6 & -30.040 & -27.774 \\
\hline 7 & -29.120 & -28.787 \\
\hline 8 & -29.217 & -29.267 \\
\hline 9 & -28.648 & -28.694 \\
\hline 10 & -28.740 & -28.975 \\
\hline 11 & -29.416 & -29.071 \\
\hline 12 & -28.833 & -29.120 \\
\hline 13 & -28.556 & -28.511 \\
\hline 14 & -28.288 & -28.833 \\
\hline 15 & -28.027 & -29.366 \\
\hline 16 & -26.905 & -28.928 \\
\hline 17 & -28.113 & -29.169 \\
\hline 18 & -27.858 & -27.942 \\
\hline 19 & -29.416 & -29.416 \\
\hline 20 & -28.648 & -28.648 \\
\hline 21 & -28.288 & -27.368 \\
\hline 22 & -28.376 & -28.556 \\
\hline 23 & -28.556 & -28.740 \\
\hline 24 & -28.928 & -29.071 \\
\hline 25 & -28.288 & -27.691 \\
\hline 26 & -28.833 & -28.556 \\
\hline 27 & -28.648 & -27.250 \\
\hline 28 & -28.376 & -28.787 \\
\hline 29 & -28.833 & -28.511 \\
\hline 30 & -27.448 & -28.511 \\
\hline 31 & -27.289 & -29.267 \\
\hline 32 & -29.316 & -27.488 \\
\hline 33 & -27.858 & -28.694 \\
\hline 34 & -27.368 & -28.975 \\
\hline 35 & -29.217 & -28.511 \\
\hline 36 & -27.528 & -28.156 \\
\hline 37 & -29.316 & -28.070 \\
\hline
\end{tabular}




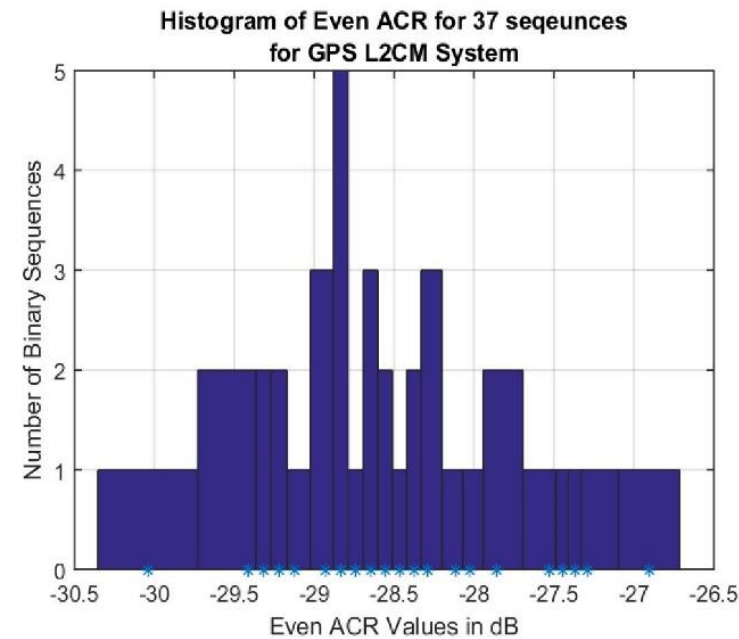

Figure 2. Histogram of maximum off peak even ACR for 37 GPS L2 CM sequences

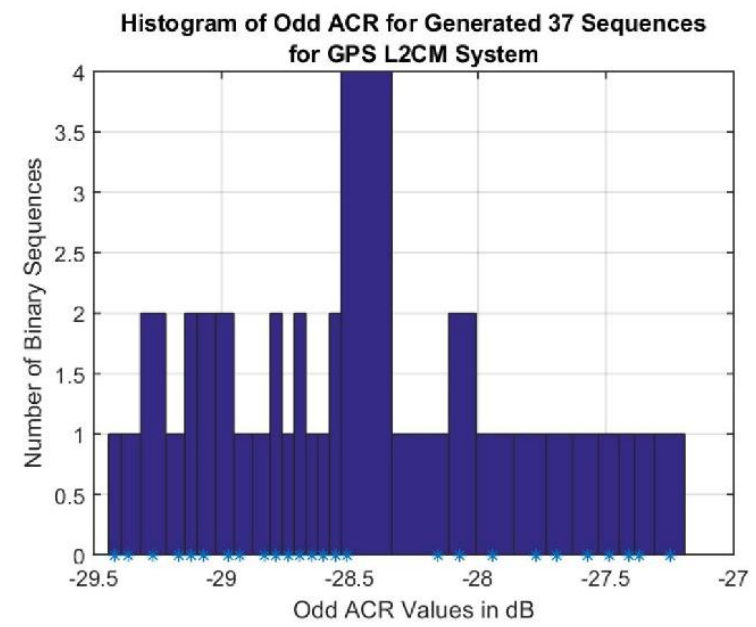

Figure 3. Histogram of maximum off peak odd ACR for 37 GPS L2 CM sequences

\subsection{Even and odd CCR properties of GPS L2 CM sequences}

Investigation is also carried out for even and odd CCR properties. The histogram of results of all 666 combinations of pairwise maximum even CCR values and pairwise maximum odd even CCR values are plotted in Figure 4 and Figure 5 respectively. From Figure 4 and Figure 5, the even and odd CCR values are found to be multiple valued because of truncation. Also in Figure 4, it is found that the maximum pairwise even CCR value is $-25.39 \mathrm{~dB}$ and the occurrences of pairwise even CCR values are found to be populated near $28.5 \mathrm{~dB}$. In Figure 5, it is found that the maximum pairwise odd CCR value is $-26.32 \mathrm{~dB}$ and the occurrences of pairwise odd CCR values are found to be populated near $-28.5 \mathrm{~dB}$.

From the correlation results following observations are made

- The odd correlation and even correlation functions of the GPS L2 CM sequences are multiple valued due to truncation of LFSR sequences.

- The GPS L2 CM Binary sequences are found to have better odd correlation performance. The odd ACR and odd CCR values are found to be better than the even ACR and even CCR values by $0.34 \mathrm{~dB}$ and $0.93 \mathrm{~dB}$ respectively. It is to be noted that the GPS L1 C/A PRN code designed during the first generation of GPS is found to have better even correlation properties when compared to odd correlation properties [18].

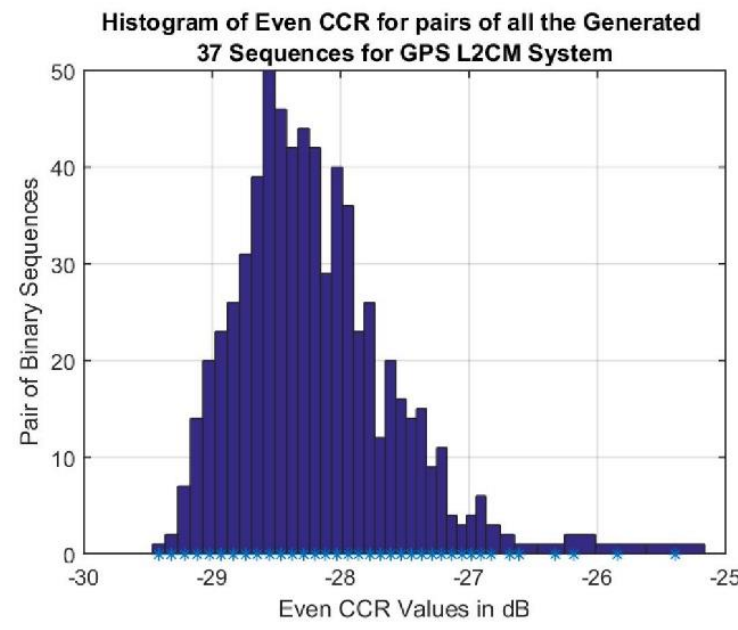

Figure 4. Histogram of pairwise maximum even CCR of all 666 combinations for 37 GPS L2 CM sequences

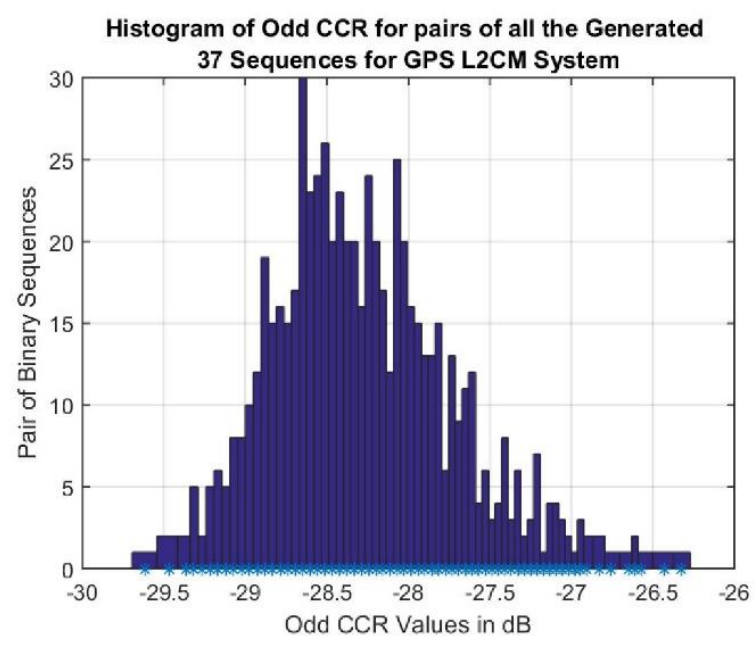

Figure 5. Histogram of pairwise maximum odd CCR of all 666 combinations for 37 GPS L2 CM sequences

\subsection{Linear Complexity properties of GPS L2 CM sequences}

Linear complexity (LC) values are computed for all 37 sequences using Berlekamp - Massey algorithm [16] and it is found to be 27 . In the next section a method is proposed to generate binary sequences having better properties than GPS L2 CM sequences.

\section{PROPOSED METHOD OF GENRATING CHAOTIC BINARY SEQUENCE}

Proposed method [18] for deriving binary sequences from chaotic sequences is shown in Figure 6.

Due to its simplicity Logistic map is the commonly used chaotic map [19-21]. This work considered chaotic sequences generated by Logistic Map defined by the equation [21]

$$
\mathrm{S}_{\mathrm{k}+1}=\mathrm{BS}_{\mathrm{k}}\left(1-\mathrm{S}_{\mathrm{k}}\right)
$$


$\mathrm{S}_{\mathrm{k}}$ is real valued chaotic output with bound $0<\mathrm{S}_{\mathrm{k}}<1, \mathrm{~B}$ is bifurcation factor. The bifurcation factor is in the range $3.57<$ $\mathrm{B}<4$ and will always ensure that the output from this system is chaotic in nature.

In the proposed method the real valued chaotic sequences are converted to binary sequences governed by the following equation [18]

$$
\mathrm{Y}_{\mathrm{k}}=\left\lfloor\left(\mathrm{S}_{\mathrm{k}}\right) * \mathrm{E}\right\rfloor \bmod (\mathrm{G})
$$

Using Eq. (6), iteratively real valued infinite sequence $\left\{S_{k}\right\}$, $\mathrm{k}=0,1,2, \ldots$, is generated. Each real valued sequence $\mathrm{S}_{\mathrm{k}}$ is multiplied by a large integer $\mathrm{E}$ and modulo operation is performed over another integer $G$ by selecting $G$ and $E$ as $G<$ E. As shown in Figure 6, the integer part $\mathrm{Q}_{\mathrm{k}}$ is considered and fraction is rejected. Resulting integer $Y_{k}$ is mapped to binary directly to obtain output sequence $\mathrm{Pk}$.

The binary sequences generated using the proposed method are investigated for correlation and linear complexity properties in the next section.

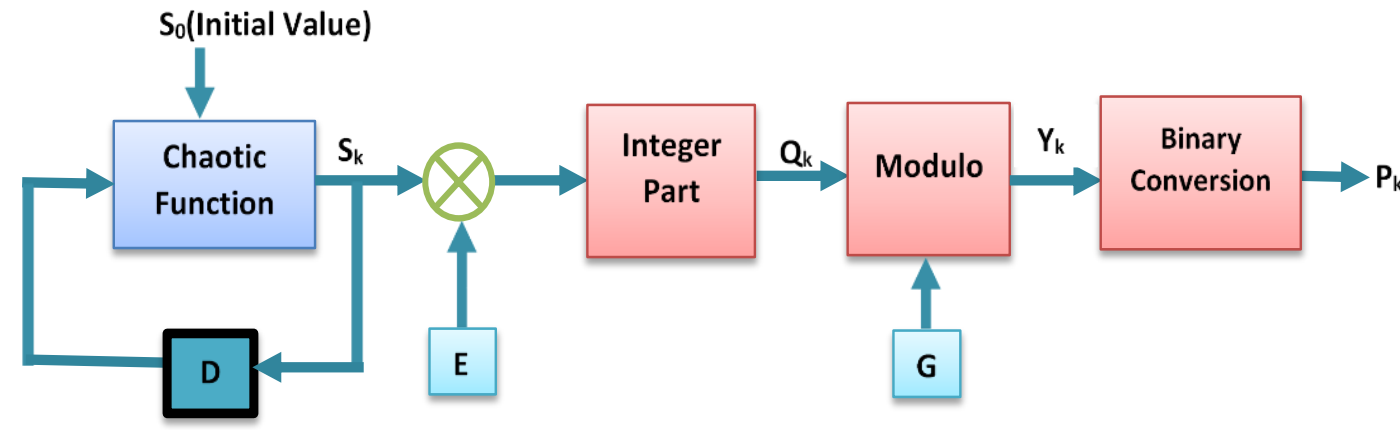

Figure 6. Proposed method for generation of binary sequences using chaotic function for GNSS applications

\section{CORRELATION AND LINEAR COMPLEXITY PROPERTIES OF THE PROPOSED BINARY SEQUENCES}

A set of 100 binary sequences of length 10230 bits were generated from the proposed method by selecting the initial values randomly and setting integer $\mathrm{G}=4, \mathrm{~B}=3.99$ and $\mathrm{E}=32767$. MATLAB simulations are performed to obtain 47 binary sequences from the proposed method and the initial values of all the 47 binary sequences are listed in Table 3.

Table 3. Initial values for generation of proposed binary sequences

\begin{tabular}{cc}
\hline $\begin{array}{c}\text { Binary } \\
\text { Seqeunce } \\
\text { Number }\end{array}$ & Initial Value \\
1 & 0.656560157622049 \\
2 & 0.379428069773115 \\
3 & 0.956473170430473 \\
4 & 0.837323305625539 \\
5 & 0.186769582443968 \\
6 & 0.917937385063156 \\
7 & 0.449003245887149 \\
8 & 0.317813959862539 \\
9 & 0.210370839512595 \\
10 & 0.421562177994972 \\
11 & 0.115709453804408 \\
12 & 0.313397650853574 \\
13 & 0.452906953664362 \\
14 & 0.707796016680767 \\
15 & 0.354081569750622 \\
16 & 0.500947441246977 \\
17 & 0.353750830722854 \\
18 & 0.563401008883089 \\
19 & 0.656157553061328 \\
20 & 0.326484624595631 \\
21 & 0.682479882613947
\end{tabular}

\begin{tabular}{l}
0.248820030659104 \\
0.677767695635484 \\
0.365907196587940 \\
0.479347941435340 \\
0.576065641236740 \\
0.216510493002707 \\
0.929057543334895 \\
0.832411734145950 \\
0.651115097100317 \\
0.682275215512988 \\
0.435351966932894 \\
0.148961992745456 \\
0.305386520123207 \\
0.682400839938918 \\
0.685798293358962 \\
0.547081326731316 \\
0.903628961195317 \\
0.784678357417467 \\
0.790692597585520 \\
0.974153022080358 \\
0.725750333188450 \\
0.686225815491298 \\
0.735791042001489 \\
0.892298117955924 \\
0.656560157622049 \\
0.379428069773115 \\
\hline
\end{tabular}

\subsection{Even and odd ACR properties of proposed sequences}

Investigation is carried out for even and odd ACR properties. The results of maximum off peak even ACR and maximum off peak odd ACR values are tabulated in Table 4. From Table 4, it is found that maximum even ACR for the set of 47 sequences is $-29.02 \mathrm{~dB}$ and the maximum odd ACR is found to be -27.28 $\mathrm{dB}$. The histogram of off peak even ACR and the off peak odd ACR is shown in Figure 7 and Figure 8 respectively. From the Figure 7, it is found that 32 sequences out of 47 sequences are having off peak maximum even ACR in the range $-29 \mathrm{~dB}$ to - 
$29.2 \mathrm{~dB}$. From the Figure 8, it is found that more than 9 sequences out of 47 sequences are having off peak odd ACR value better than $-28.5 \mathrm{~dB}$.

Table 4. Off peak maximum even ACR value and off peak maximum odd ACR value of the proposed binary seqeunces

\begin{tabular}{|c|c|c|}
\hline $\begin{array}{l}\text { Proposed } \\
\text { Binary }\end{array}$ & $\begin{array}{c}\text { Off Peak } \\
\text { Maximum even }\end{array}$ & $\begin{array}{l}\text { Off Peak Maximum } \\
\text { odd ACR Value }\end{array}$ \\
\hline Sequence & ACR Value & \\
\hline Number & & \\
\hline 1 & -29.217 & -27.650 \\
\hline 2 & -29.023 & -29.120 \\
\hline 3 & -29.416 & -29.169 \\
\hline 4 & -29.120 & -27.733 \\
\hline 5 & -29.723 & -29.120 \\
\hline 6 & -29.120 & -28.421 \\
\hline 7 & -29.416 & -28.648 \\
\hline 8 & -29.517 & -28.740 \\
\hline 9 & -29.023 & -27.984 \\
\hline 10 & -29.416 & -28.648 \\
\hline 11 & -29.023 & -28.556 \\
\hline 12 & -29.619 & -27.691 \\
\hline 13 & -29.217 & -28.833 \\
\hline 14 & -29.120 & -28.421 \\
\hline 15 & -29.120 & -29.023 \\
\hline 16 & -29.723 & -29.416 \\
\hline 17 & -29.120 & -29.619 \\
\hline 18 & -29.023 & -28.648 \\
\hline 19 & -29.217 & -27.528 \\
\hline 20 & -29.316 & -28.200 \\
\hline 21 & -29.217 & -28.975 \\
\hline 22 & -29.023 & -29.023 \\
\hline 23 & -29.120 & -28.648 \\
\hline 24 & -29.316 & -29.416 \\
\hline 25 & -29.517 & -28.027 \\
\hline 26 & -29.120 & -28.833 \\
\hline 27 & -29.316 & -28.376 \\
\hline 28 & -29.217 & -29.466 \\
\hline 29 & -29.316 & -28.027 \\
\hline 30 & -29.217 & -28.466 \\
\hline 31 & -29.023 & -27.488 \\
\hline 32 & -29.120 & -28.376 \\
\hline 33 & -29.120 & -29.120 \\
\hline 34 & -29.619 & -28.556 \\
\hline 35 & -29.217 & -28.602 \\
\hline 36 & -29.023 & -28.288 \\
\hline 37 & -29.217 & -28.511 \\
\hline 38 & -29.416 & -29.120 \\
\hline 39 & -29.120 & -28.200 \\
\hline 40 & -29.023 & -29.120 \\
\hline 41 & -29.023 & -27.528 \\
\hline 42 & -29.217 & -28.511 \\
\hline 43 & -29.023 & -27.984 \\
\hline 44 & -29.316 & -28.556 \\
\hline 45 & -29.933 & -27.569 \\
\hline 46 & -29.120 & -28.156 \\
\hline 47 & -29.217 & -28.511 \\
\hline
\end{tabular}

\subsection{Even and odd CCR properties of proposed sequences}

Investigation is carried out for even and odd CCR properties. The histogram of results of all 1081 combinations of pairwise maximum even CCR values and pairwise maximum odd even CCR values are plotted in Figure 9 and Figure 10 respectively. From the Figure 9, it is found that the maximum pairwise even CCR value is $-26.01 \mathrm{~dB}$ and the occurrences of pairwise even CCR values are found to be populated near $-28.5 \mathrm{~dB}$. From Figure 9 , it is found that the maximum pairwise odd CCR value is $-26.32 \mathrm{~dB}$ and the occurrences of pairwise odd CCR values are found to be populated near $-28.5 \mathrm{~dB}$.

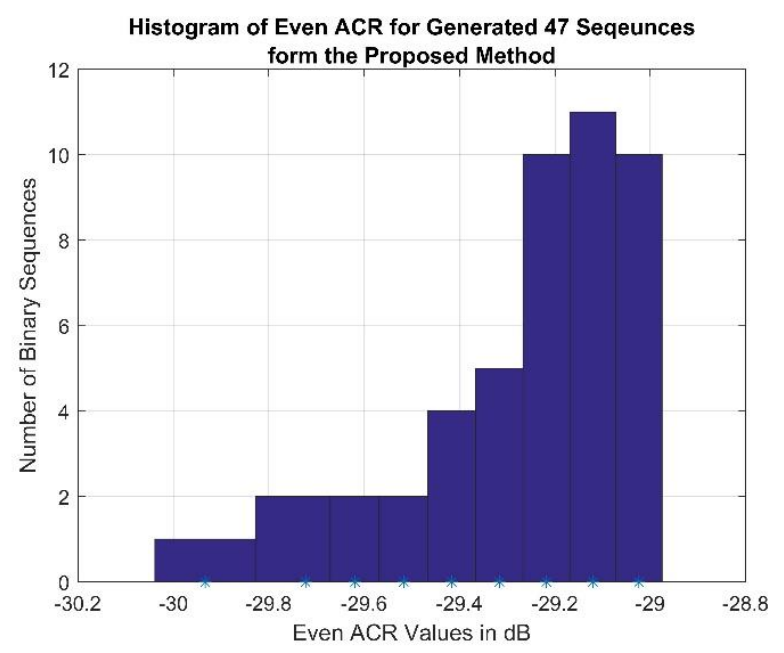

Figure 7. Histogram of maximum off peak even ACR for 47 proposed binary sequences

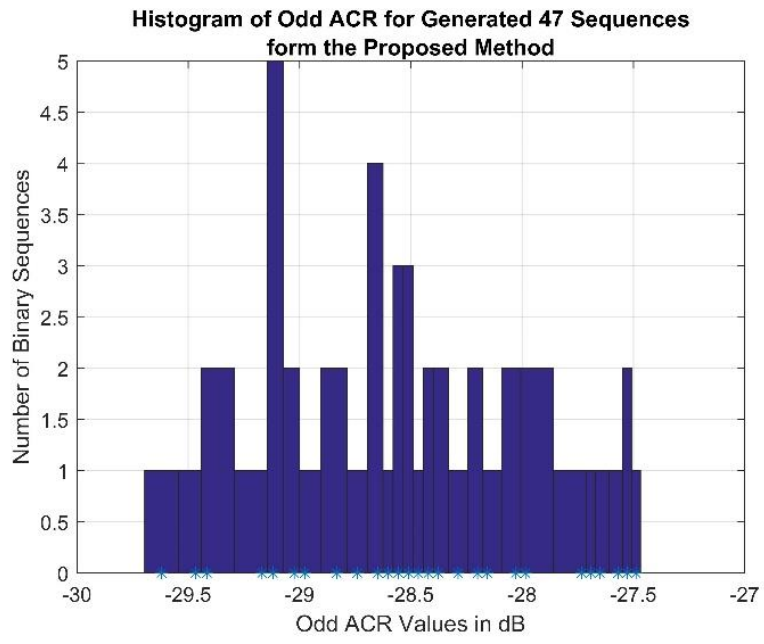

Figure 8. Histogram of maximum off peak odd ACR for 47 proposed binary sequences

The histogram results of all 1081 combinations of pairwise maximum even CCR and pairwise maximum odd CCR for a set of proposed 47 binary sequences are as shown in Figure 9 and Figure 10 respectively. From the histogram it is observed that for a set of 47 binary sequences of length 10230 bits, the maximum pairwise even cross correlation is $-26.01 \mathrm{~dB}$ and maximum pairwise odd cross correlation is found to be $-26.32 \mathrm{~dB}$.

Table 5 provides comparison of the correlation properties of the proposed sequences and GPS L2 CM sequences.

Table 5. Comparison of Correlation properties of proposed sequences and GPS L2 CM sequences

\begin{tabular}{cccc}
\hline S1 & Property & $\begin{array}{c}\text { GPS L2 } \\
\text { CM }\end{array}$ & $\begin{array}{c}\text { Proposed } \\
\text { Sequences }\end{array}$ \\
& & sequences & \\
1 & Even ACR & $-29.02 \mathrm{~dB}$ & $-26.90 \mathrm{~dB}$ \\
2 & Odd ACR & $-27.48 \mathrm{~dB}$ & $-27.24 \mathrm{~dB}$ \\
3 & Even CCR & $-26.21 \mathrm{~dB}$ & $-25.39 \mathrm{~dB}$ \\
4 & Odd CCR & $-26.32 \mathrm{~dB}$ & $-26.32 \mathrm{~dB}$ \\
\hline
\end{tabular}


From the Table 5 following observations are made

- Proposed binary sequences outperform the GPS L2 CM sequences in maximum off peak even ACR by $2.12 \mathrm{~dB}$.

- Proposed set of binary sequences are found to have better even CCR by $0.82 \mathrm{~dB}$ as compared to the GPS L2 CM sequences.

- Proposed set of binary sequences are found to be having better maximum off peak odd ACR by $0.24 \mathrm{~dB}$.

- Odd CCR property is same for both the binary sequences.

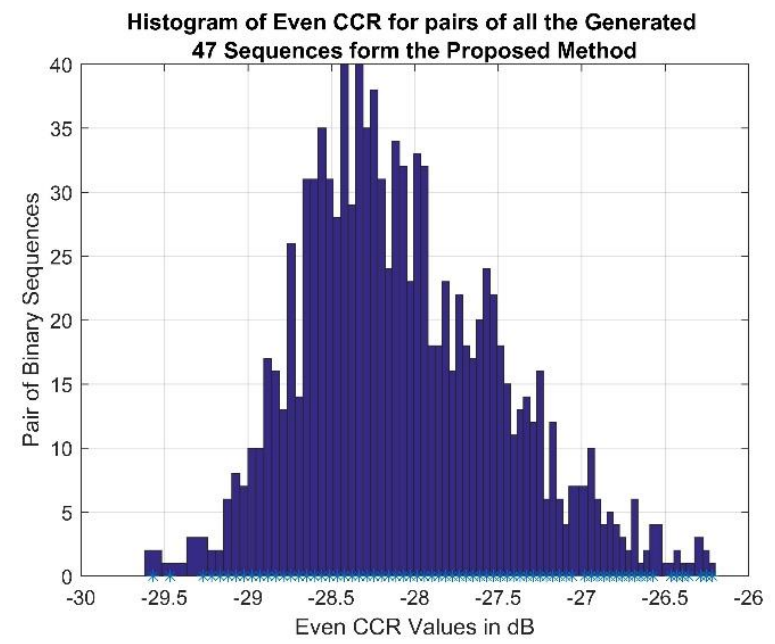

Figure 9. Histogram of pairwise maximum even CCR of all 1081 combinations for 47 proposed binary sequences

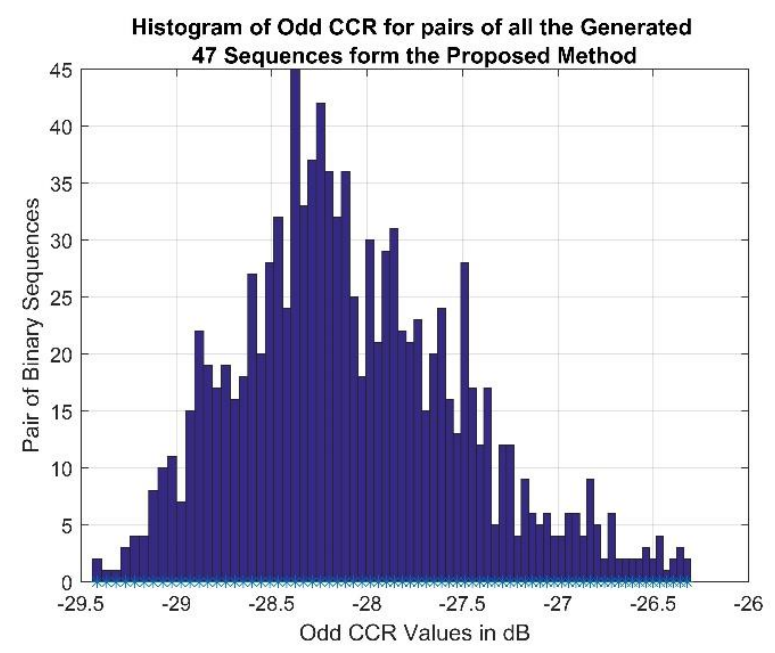

Figure 10. Histogram of pairwise maximum odd CCR of all 1081 combinations for 47 proposed binary sequences

\subsection{Linear Complexity properties of proposed sequences}

The Linear complexity (LC) property profile of the generated 47 binary sequences of length 10230 bits are computed using Berleykamp- Massey Algorithm [16]. In Table 6, the values of LC for all the 47 proposed binary sequences along with their initial values are tabulated. It is found from Table 4 that the linear complexity for 10230 bits binary sequences proposed is $5115 \pm 3$. Security of the proposed sequences is excellent as compared to GPS L2 CM sequences.

The Linear complexity profile for proposed binary sequences is as shown in Figure 11. The dotted line represents the expected linear complexity value of 5115 for ideal random sequences of length 10230 bits. The continuous line represents the Linear Complexity of the Proposed 47 binary sequences. From the Figure 11 it is observed that the proposed binary sequences possess the near ideal linear complexity property.

Table 6. Initial values for generation of proposed binary sequences and linear complexity property of proposed binary sequences

\begin{tabular}{|c|c|c|}
\hline $\begin{array}{c}\text { Binary } \\
\text { Seqeunce } \\
\text { Number }\end{array}$ & Initial Value $S_{0}$ & $\begin{array}{c}\text { LC value } \\
\text { of } \\
\text { generated } \\
\text { sequence }\end{array}$ \\
\hline 1 & 0.656560157622049 & 5113 \\
\hline 2 & 0.379428069773115 & 5116 \\
\hline 3 & 0.956473170430473 & 5116 \\
\hline 4 & 0.837323305625539 & 5116 \\
\hline 5 & 0.186769582443968 & 5115 \\
\hline 6 & 0.917937385063156 & 5116 \\
\hline 7 & 0.449003245887149 & 5115 \\
\hline 8 & 0.317813959862539 & 5115 \\
\hline 9 & 0.210370839512595 & 5116 \\
\hline 10 & 0.421562177994972 & 5116 \\
\hline 11 & 0.115709453804408 & 5117 \\
\hline 12 & 0.313397650853574 & 5115 \\
\hline 13 & 0.452906953664362 & 5116 \\
\hline 14 & 0.707796016680767 & 5114 \\
\hline 15 & 0.354081569750622 & 5116 \\
\hline 16 & 0.500947441246977 & 5115 \\
\hline 17 & 0.353750830722854 & 5115 \\
\hline 18 & 0.563401008883089 & 5116 \\
\hline 19 & 0.656157553061328 & 5116 \\
\hline 20 & 0.326484624595631 & 5115 \\
\hline 21 & 0.682479882613947 & 5115 \\
\hline 22 & 0.248820030659104 & 5115 \\
\hline 23 & 0.677767695635484 & 5115 \\
\hline 24 & 0.365907196587940 & 5115 \\
\hline 25 & 0.479347941435340 & 5116 \\
\hline 26 & 0.576065641236740 & 5115 \\
\hline 27 & 0.216510493002707 & 5114 \\
\hline 28 & 0.929057543334895 & 5115 \\
\hline 29 & 0.832411734145950 & 5115 \\
\hline 30 & 0.651115097100317 & 5115 \\
\hline 31 & 0.682275215512988 & 5115 \\
\hline 32 & 0.435351966932894 & 5115 \\
\hline 33 & 0.148961992745456 & 5115 \\
\hline 34 & 0.305386520123207 & 5116 \\
\hline 35 & 0.682400839938918 & 5115 \\
\hline 36 & 0.685798293358962 & 5115 \\
\hline 37 & 0.547081326731316 & 5114 \\
\hline 38 & 0.903628961195317 & 5115 \\
\hline 39 & 0.784678357417467 & 5113 \\
\hline 40 & 0.790692597585520 & 5116 \\
\hline 41 & 0.974153022080358 & 5115 \\
\hline 42 & 0.725750333188450 & 5114 \\
\hline 43 & 0.686225815491298 & 5115 \\
\hline 44 & 0.735791042001489 & 5112 \\
\hline 45 & 0.892298117955924 & 5116 \\
\hline 46 & 0.656560157622049 & 5116 \\
\hline 47 & 0.379428069773115 & 5115 \\
\hline
\end{tabular}




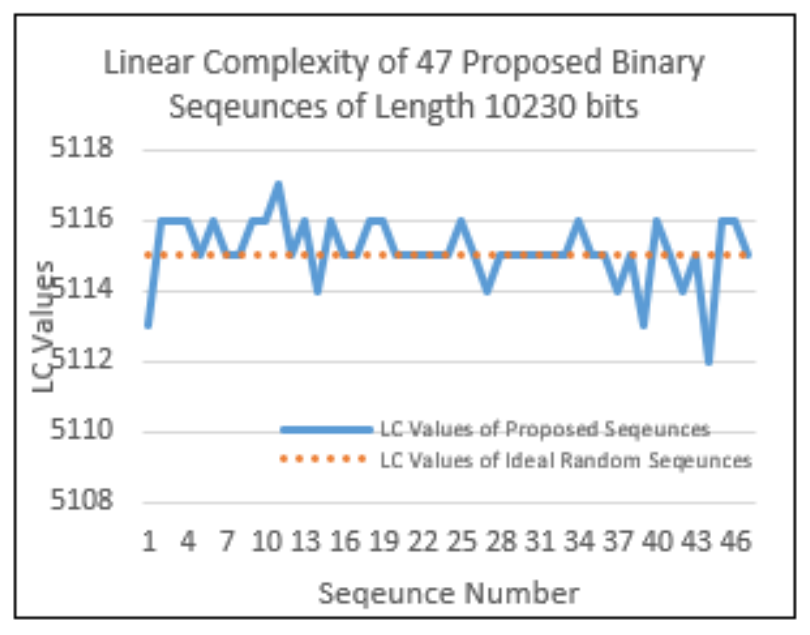

Figure 11. Linear Complexity profile of proposed set of 47 binary sequences of length 10230 bits in comparison of ideal random sequences Linear Complexity (LC) profile

\section{CONCLUSION}

In this work, a set of 37 GPS L2 CM binary sequences are generated. The odd correlation, even correlation and the linear complexity properties are analyzed for GPS L2 CM binary sequences. It is observed that the GPS L2 CM Sequences are obtained by truncating very long LFSR sequence and hence the correlation properties are degraded due to truncation. It is also found that the maximum off peak even ACR value is $26.90 \mathrm{~dB}$, maximum of peak odd ACR value is $-27.24 \mathrm{~dB}$, maximum pairwise even $\mathrm{CCR}$ value is $-25.39 \mathrm{~dB}$, maximum pairwise odd CCR value is found to be $-26.32 \mathrm{~dB}$ and $\mathrm{LC}$ value is 27 .

A method is also proposed to generate binary sequences of length 10230 bits. A set of 47 binary sequences of length 10230 bits are generated. The generated binary sequences are analyzed for odd correlation, even correlation and linear complexity properties. For the set of 47 sequences of length 10230 bits it is found that maximum off peak even ACR value is $-29.02 \mathrm{~dB}$, maximum off peak odd ACR value is -27.24 $\mathrm{dB}$, maximum pairwise even $\mathrm{CCR}$ value is $-26.02 \mathrm{~dB}$, maximum pairwise odd CCR value is found to be $-26.32 \mathrm{~dB}$ and the $\mathrm{LC}$ is found to be $5115 \pm 3$.

It is found that the proposed sequences as compared to GPS L2 CM binary sequences are better in having maximum off peak even ACR of $2.12 \mathrm{~dB}$ greater, pairwise maximum even CCR of $0.82 \mathrm{~dB}$ grater, maximum off peak odd ACR by 0.24 $\mathrm{dB}$ higher value. The generated sequences are found to have large LC of $5115 \pm 3$ as compared to LC of 27 for GPS L2 CM binary sequences and hence provides excellent inherent security.

Due to the better correlation properties and large linear complexity the proposed sequences are suitable for use in secure GNSS Applications.

\section{REFERENCES}

[1] Wallner S., Avila-Rodriguez, J.A., Hein, G.W., Rushanan, J.J. (2007). Galileo E1 OS and GPS L1C psuedo random noise codes, requirements, generation, optimization and comparison. Proceedings of the 20th International Technical Meeting of the Satellite Division of The Institute of Navigation (ION GNSS 2007), Fort Worth, Texas, pp. 1549-1563.

[2] Stefan, W., Jose, A., Avila, R. (2011). Codes: The PRN family grows again. Inside GNSS working paper, Inside GNSS Magazine Article, 83-92.

[3] Ravichandran, P.N., Kulkarni, S.J., Vasudevamurthy, H.S., Vanitha, M. (2012). Analysis and Simulation of Pseudo Ranging Noise codes for GeoStationary Satellites and its Doppler Effect. ACEEE International Journal on Communication, 3(2): 17-23.

[4] Hein, G.W., Avila-Rodriguez, J.A., Wallner S. (2011). The DaVinci Galileo Code and others. Inside GNSS working paper, Inside GNSS Magazine Article, 62-74.

[5] Dharmappa, D., Mahalinga, V.M., Ramesh, S. (2016). A survey on binary sequence generation methods for global navigation satellite systems (GNSS). Far East Journal of Electronics and Communications, 16(4): 775-788. http://dx.doi.org/10.17654/EC016040775

[6] Gao, G.X., Akos, D., Walter, T., Enge, P. (2008). Understanding the GIOVE-B broadcast codes of the Galileo system. 2008 42nd Asilomar Conference on Signals, Systems and Computers, Pacific Grove, CA, USA, pp. 2086-2090. http://doi.org/10.1109/ACSSC.2008.5074800

[7] Psiaki, M.L., Humphreys, T.E., Mohiuddin, S., Powell, S.P., Cerruti, A.P., Kintner, P.M. (2006). Searching for Galileo. ION GNSS 19th International Technical Meeting of the Satellite Division, 26-29, Fort Worth, TX.

[8] Ramesh, S., Haribhat, K.N., Murali, R. (2010). On the linear complexity of binary sequences generated using matrix recurrence relation defined over Z4. International Journal of Distributed and Parallel Systems (IJDPS), 1(2): 68-85. http://doi.org/10.5121/ijdps.2010.1207

[9] Mandi, M.V., Haribhat, K.N., Murali, R. (2010). Generation of large set of binary sequences derived from chaotic functions with large linear complexity and good cross correlation properties. International Journal of Advanced Engineering and Applications (IJAEA), 1(1): 93-112. http://doi.org/10.5121/ijdps.2010.1107

[10] Boztas, S., Kumar, P.V. (1994). Binary sequences with Gold-like correlation but larger linear span. IEEE Transactions on Information Theory, 40(2): 532-537. http://doi.org/10.1109/18.312181

[11] Gong, G. (2002). New designs for signal sets with low cross correlation, balance property, and large linear span: GF (p) case. IEEE Transactions on Information Theory, 48(11): 2847-2867. http://doi.org/10.1109/TIT.2002.804044

[12] Zhu, Y., Tjhung, T.T., Garg, H.K. (1999). A new family of polyphase sequences for CDMA with good odd and even correlation properties. 1999 2nd IEEE Workshop on Signal Processing Advances in Wireless Communications (Cat. No.99EX304), Annapolis, MD, USA. http://doi.org/10.1109/SPAWC.1999.783053

[13] Fukumasa, H., Kohno, R., Imai, H. (1994). Design of pseudonoise sequences with good odd and even correlation properties for DS/CDMA. IEEE Journal on Selected Areas in Communications, 12(5): 828-836. http://doi.org/10.1109/49.298056

[14] Dudkov, A., Ipatov, V.P. (2005). Signature-interleaved DS CDMA: Controlling odd correlation peaks. 2005 IEEE 16th International Symposium on Personal, Indoor and Mobile Radio Communications, Berlin, Germany. https://doi.org/10.1109/PIMRC.2005.1651899 
[15] Sarwate, D.V., Pursley, M.B. (1980). Crosscorrelation properties of pseudorandom and related sequences. Proceedings of IEEE, 68(5): 593-619. https://doi.org/10.1109/PROC.1980.11697

[16] Massey, J.L. (1969). Shift register synthesis and BCH decoding. IEEE Trans. on Information Theory, 15(1): 122-127. https://doi.org/10.1109/TIT.1969.1054260

[17] GPS Interface Control Document. (2016). Interface Specification IS-GPS-200 Revision H, IRN003 (IS-GPS200H), 1-236.

[18] Dharmappa, D., Mandi, M.V., Ramesh, S. (2017). Generation of binary sequences having better odd correlation and linear complexity property compared to gold codes for use in global navigation satellites systems (GNSS) applications. 2017 International Conference on Current Trends in Computer, Electrical, Electronics and Communication (CTCEEC), Mysore, India, pp. 155-160. https://doi.org/10.1109/CTCEEC.2017.8455026

[19] Heidari-Bateni, G., McGillem, C.D. (1994). A Chaotic Direct-sequence spread-spectrum communication system. IEEE Transactions on Communications, 42(234): 1524-1527. https://doi.org/10.1109/10.1109/TCOMM.1994.582834

[20] Ling, G., Li, S.Q. (2000). Chaotic spreading sequences with multiple access performance better than random sequences. IEEE Transactions on Circuits and Systems-

I: Fundamental Theory and Applications, 47(3): 394-397. https://doi.org/10.1109/81.841922

[21] May, R.M. (1976). Simple mathematical models with very complicated dynamics. Nature, 261: 459-474. https://doi.org/10.1038/261459a0

\section{NOMENCLATURE}

$\mathrm{S}_{\mathrm{k}}$

B

E

G

$\mathrm{Y}_{\mathrm{k}}$

\section{Subscripts}

$\mathrm{k}$

real valued chaotic output. This is a dimension less quantity

bifurcation factor for chaotic logistic map. This is a dimensionless quantity large positive integer, dimensionless quantity

positive integer, dimensionless quantity integer valued output from the chotic generator. This is a dimension less quantity

$\mathrm{k}^{\text {th }}$ symbol output from the proposed sequence generator binary sequence $\mathrm{x}$ binary sequence $y$ 